\title{
Time series distances measures to analyze in-air signatures to authenticate users on mobile phones
}

\author{
Javier Guerra-Casanova, Carmen Sánchez Ávila, Gonzalo Bailador, Alberto de-Santos-Sierra \\ Centro de Domótica Integral \\ Universidad Politécnica de Madrid \\ Campus de Montegancedo \\ 28223 Pozuelo de Alarcón, Madrid, SPAIN \\ Email:[jguerra, csa, gbailador, alberto]@cedint.upm.es
}

\begin{abstract}
Improving the security of mobile phones is one of the crucial points required to assure the personal information and the operations that can be performed from them. This article presents an authentication procedure consisting of verifying the identity of people by making a signature in the air while holding the mobile phone. Different temporal distance algorithms have been proposed and evaluated through a database of 50 people making their signatures in the air and 6 people trying to forge each of them by studying their records. Approaches based on DTW have obtained better EER results than those based on LCS $(2.80 \%$ against $3.34 \%$ ). Besides, different signal normalization methods have been evaluated not finding any with better EER results that when no normalization has carried out.
\end{abstract}

Index Terms - In-air signature, time series distances, Longest Common Subsequence, Dynamic Time Warping, mobile biometrics, gestures.

\section{INTRODUCTION}

At present, mobile phones are very powerful devices where people may perform a lot of operations that may require the verification of their identity, such as e-commerce applications. In addition to this, mobile phones store a lot of personal information that should not be revealed to anyone except the owner of the phone. Therefore, mobile phones are nowadays very useful devices, but they require security in order to keep safe the information and the operations performed with them. In this context, biometrics could help complementing or substituting PIN-code security.

There are many works including biometric authentication techniques in mobile devices to enhance their security [1]. Many different biometric characteristics are used in this aim. In [2] and [3] people are authenticated by the recognition of their face through the camera of a mobile phone. In [4] and [5] authentication is provided by means of iris and in [6] the characteristic biometric features are voice and fingerprint. In addition to this, in [7] users are recognized by keystroke analysis.

This article is based on authenticating people by making their signature in the air while holding their mobile phone in their hand. This biometric technique has similarities with handwritten signature as well as gesture recognition approaches.

Users enroll in their mobile phone by making three times their signature in the air. Then some operations are performed in order to obtain some values that are included in the user template. The verification of the identity of the user will be achieved when he/she repeats his/her in-air signature accurately.

The only requirement of mobile phones to be valid to be used in this authentication technique is that they must embed an accelerometer. In this manner, when users make their signature in the air, the accelerometers would obtain the acceleration values of the movement at a sampling rate of 50 $\mathrm{Hz}$. The analysis of these temporal signals is carried out using algorithms that calculate temporal distances. Actually, in this article, different approaches of Longest Common Subsequence and Dynamic Time Warping algorithms are proposed and evaluated.

According to this, Section II introduces some of the related work this article is based on. Then, Section III explains the temporal distance analysis approach used to compare different acceleration signals and also the overall authentication system. In addition to this, the different normalization techniques evaluated in this article are also described in this Section. Next, the experimental procedure of this article is detailed in Section IV containing the characteristics of the database and the metrics used to evaluate all the approaches of the article. The results of these experiments are shown in Section V. Finally, the conclusions and future work are presented in Section VI.

\section{RELATED WORK}

In this article, two temporal series distances (Longest Common Subsequence, LCS and Dynamic Time Warping, DTW) are studied to analyze the acceleration signals when making an in-air signature holding a mobile device. Both signals are based on dynamic programming [8], as a method to solve the optimal alignment between two signals.

Dynamic Time Warping [9] DTW finds the optimal alignment by minimizing the Euclidean distance between two signals. Many works utilizing DTW algorithms to solve pattern recognition problems have been carried out [10].

This algorithm has many different approaches, such as Derivative DTW [11] or Fast DTW [12].

Furthermore, Dynamic Time Warping has been also previously used in biometrics, specially in handwritten signature 
verification [13], [14] as well as in gesture recognition [15], [16].

On the other hand, Longest Common Subsequence algorithm looks for the optimal alignment by maximizing the length of the common subsequence of two signals [17], [18]. There are also quite different approaches of applying LCS algorithms, such a constrained LCS [19] or Fast LCS [20].

As well as DTW, LCS has been also applied in the biometric [21] and gesture recognition fields [22], [23].

Besides, the authors have previously developed some works analyzing in-air signatures by means of a generalized LCS algorithm [24] with different modifications [25] and different punctuation scores [26].

\section{DESCRIPTION OF THE IN AIR SIGNATURE AUTHENTICATION SYSTEM}

This section presents the following subsections:

- Normalization techniques, where the different normalization approaches studied in this article are explained in order to evaluate to what extent is useful to normalize the acceleration signals before analyzing them.

- Temporal distance analysis approach, where it is described how two acceleration signals are compared and how the score that quantifies the differences of the signals is obtained. All the algorithms assessed in the article are described.

- Comparison of repetitions of in-air signatures, where how to compare two signature samples is detailed. In the previous subsection, the analysis was carried out only through two signals. However, as each signature is made up of three signals (one from each axis), how to fuse the scores of all of them is presented in this Section.

- Complete verification system, where how the overall system works is described.

\section{A. Normalization techniques}

The accelerometers utilized in this article provided the acceleration value at a sampling rate of $50 \mathrm{~Hz}$ and within a range of $[-2.5 \mathrm{~g}, 2.5 \mathrm{~g}]$. According to this, this article evaluates whether the performance of different algorithms improves or worsens when the acceleration signals have been previously normalized.

Four different normalization techniques are assessed:

- No normalization. The signals obtained for the accelerometer are no modified at all.

- Maximum normalization. Each acceleration signal is divided by 2.5 in order to normalize all the signals in the range $[-1,1]$.

- Maximum on-signal normalization. Each acceleration signal is divided by its maximum absolute value.

- Maximum-average normalization. Each acceleration signal is first subtracted by the average of the signal, and afterwards, divided by its maximum absolute value. According to this, the signals are centered around 0 and belong to the range $[-1,-1]$
Results for each normalization procedure and each temporal distance are presented in Section V.

\section{B. Temporal distance analysis approach}

A pair of acceleration signals are compared utilizing the algorithms described below. The aim of these algorithms is provide a metric in order to quantify how different are the signals. From this metric, it can be deducted whether the signals come from two repetitions of a gesture made by the same user, since it is assumed and proved later only authentic users are able to repeat accurately their signatures in the air.

Different algorithms to calculate distances of temporal signals are presented and evaluated. These algorithms are divided in two approaches:

- In the first two algorithms, the metric is calculated from the values of a score matrix filled by a equation. The first one tries to maximize the similarity of the signals (LCS) whereas the second minimizes their differences (DTW).

- The second two algorithms (ELCS and EDTW) utilized the previous algorithms to align the signals, and then the metric value is calculated from the Euclidean distance of the already aligned signals.

1) Longest Common Subsequence: The Longest Common Subsequence (LCS) algorithm looks for the longest subsequence in common of the two sequences that are compared. This algorithm finds the optimal alignment of two sequences with less edition distance, which corresponds to the longest common subsequence of both signals. The length of the LCS is the metric provided by the algorithm. The longest the LCS the more similar the sequences in comparison.

Different approaches based on Longest Common Subsequence algorithm have been developed to make this algorithm appropriate for acceleration signals, in which each value of the signal may have infinite values, as the accelerations when making a movement in the air are continuous signals. (In fact, because of sampling, the possible values of accelerations are not infinite but very large).

According to this, it is not trivial to define when two acceleration signal points have the same value. Different distances have been tried in order to describe points belonging to the LCS of two acceleration signals:

- Absolute value: Two points of the signals are considered the same when $\left|a_{i}-b_{j}\right|<\epsilon$.

- Euclidean value: Two points of the signals belong to a common subsequence if $\left(a_{i}-b_{j}\right)^{2}<\epsilon$.

In addition to this, a different approach has been developed, including the distance to describe when two points belongs to a common subsequence inside the LCS algorithm.

Therefore, the Longest Common Subsequence of signals $v(i)$ and $w(j)$ are obtained following Eq.1:

$$
s_{i, j}=\max \left\{\begin{array}{c}
s_{i, j-1}+h \\
s_{i-1, j-1}+\Delta \\
s_{i-i, j}+h
\end{array}\right.
$$

In this Equation, $\Delta$ is a function that calculates how different are the points $v_{i}$ and $w_{j}$ following Eq.2: 


$$
\Delta=-\frac{\left(v_{i}-w_{j}\right)^{2}}{2 \sigma}
$$

In Eq. 1 and 2, there are two constants whose values have already been optimized in other works [26].

The approach based on $\Delta$ distance provides better performance results than the others, so it is the one whose results are presented. In this algorithm, the value $s\left(L_{i}, L_{j}\right)$ is the equivalent to the Longest Common Subsequence metric when sequences with finite possible values are compared. ( $L_{i}$ and $L_{j}$ are the length of the signals which are compared).

The higher the value $s\left(L_{i}, L_{j}\right)$, the more similar the signals and vice versa.

2) Dynamic Time Warping (DTW): Dynamic Time Warping is also an algorithm that provides a metric of the differences of two temporal signals. In this case, DTW tries to obtain the alignment of the signals with minimal difference between them.

The Dynamic Time Warping of signals $v(i)$ and $w(j)$ consists on calculating the score matrix $S$ following Eq.3:

$$
s_{i, j}=\min \left\{\begin{array}{c}
s_{i, j-1}+d \\
s_{i-1, j-1}+d \\
s_{i-i, j}+d
\end{array}\right.
$$

In this equation, $d$ is the Euclidean distance of the points of the signals corresponding to the value of the score matrix $s_{i, j}$. It is obtained following Eq.4:

$$
d=\left(v_{i}-w_{j}\right)^{2}
$$

The value $s\left(L_{i}, L_{j}\right)$ of the score matrix $S$ is the metric of the algorithm. In this case, and opposite to LCS, the lower $s\left(L_{i}, L_{j}\right)$ the more similar two signals are.

In addition to this, the resulting value of the algorithm has been divided by the average of the length of the signals.

3) Euclidean Longest Common Subsequence (ELCS): The LCS algorithm can be also be utilized to align the signals in comparison. From the score matrix $S$, the optimal alignment of both signals can be effortless deducted.

When filling matrix $S$, a second matrix $T$ should be filled at the same time, storing whether the maximum value of Eq.1 has been obtained from the first expression (horizontal movement), the second (diagonal) or the third (vertical).

The optimal path is obtained following the movements in $T$ to join $T\left(L_{i}, L_{j}\right)$ and $T(1,1)$. A vertical movement in the matrix means repeating the corresponding value of $v(i)$ (deletion in $v$ ) whereas an horizontal implies repeating the value of $w(j)$.

Once the alignment is made, Euclidean distance of the aligned signals is calculated as follows in Eq.5:

$$
E=\sum_{k=1}^{L^{\prime}}\left(v_{k}^{\prime}-w_{k}^{\prime}\right)^{2}
$$

In Eq.5, $v^{\prime}$ and $w^{\prime}$ stand for the aligned signals, and $L^{\prime}$ for the average of the lengths of the signals after the alignment.
Notice that when aligning, the length of the signals is modified because of the repetitions of the values. Indeed, the more similar the signals the longest the common subsequence, so in very similar signals very few corrections are needed and the length of the aligned signals would keep close to the initial signals. This behaviour is the opposite when signals are quite different: a very large number of correction should be made to align the signals and the length of the signals would increase as well.

The resulting value of this approach is divided by the average of the length of the initial signals, as better performance results are obtained.

As a result of this algorithm, a comparison value is obtained. The lower it is, the more similar the signals are and vice versa.

4) Euclidean Dynamic Time Warping (EDTW): The DTW algorithm can also be used to align the signals in an equivalent manner than in ELCS. In this case, the optimal path obtained is the one where the Euclidean distance of the signals is minimal.

In spite of utilizing the final value of the path, this approach proposes to make the alignment and after that calculate the Euclidean distance following previous Eq.5.

The resulting value is also divided by the average of the length of the initial signals. The lower this value is, the more similar the signals are and vice versa.

\section{Comparison of repetitions of in-air signatures}

In the previous subsection, four different algorithms have been introduced to obtain a score to quantify how different two temporal signals are. As in-air signatures are made up of three different signals (one for each axis), different approaches might be proposed to fuse these signals or the scores obtained on a separate analysis of all of them.

In this work we propose to compare separately the signals of each axis, and then, calculate the average of the scores obtained on each comparison. Consequently, the procedure will come up with a unique score value that quantifies how similar two in-air signatures are.

\section{Complete verification system}

Users enrolling in the system repeat three times their signature in the air. Afterwards, each pair of in-air signatures is analyzed, obtaining three score values. The average of the comparison values of each pair of the three performances of gestures at enrollment is symbolize as $\mu$. This value is stored with the signals of the three repetitions of the in air signature as the template of the user.

Users already enrolled desiring to access the system should carry out once their in air signature. Then, this sample is compared with the three signatures performed at enrolling phase, obtaining three comparison values.

$\Psi$ value is calculated as the average of these scores. The score value $\Psi$ stands for the difference between the signature made at accessing phase in respect to the samples at enrolling phase. The lower it is, the most similar the performance of the signature is in relation to the template. 
If Equation 6 is accomplished (for DTW, ELCS and EDTW approaches), the user would access the system. Otherwise, he/she would be rejected. Obviously, the higher the threshold $\theta$ is, the more falsification attempts would forge the system but the less original users would be rejected, and vice versa.

$$
\frac{\Psi}{\mu}<\theta
$$

However, in LCS approach, since a high $\Psi$ means a very similar signature, the sign of the inequation to grant or deny access is different, following Eq. 7:

$$
\frac{\Psi}{\mu}>\theta
$$

\section{EXPERIMENTAL PROCEDURE}

\section{A. Database}

The experimental work of this article has been developed from a private database of in-air signatures made up of the samples of 50 people who have performed their signature in the air while holding a mobile device in their hand. This mobile device included a 3-D accelerometer programmed to obtained the acceleration values on each axis at a sampling rate of $50 \mathrm{~Hz}$.

Each user made their signature seven times in front of a video camera. From the study of these recordings, six different people tried to imitate each of the authentic in-air signatures. Each forger made 5 attempts on each real in-air signature.

Summarizing, the database consists of 250 authentic samples (50 users, 7 times) and 2100 real falsification attempts (50 users, 5 times, 6 impostors).

\section{B. Performance metric}

The performance of the algorithms previously introduced is evaluated twofold, presenting the Equal Error Rate and the Receiver Operating Characteristic (ROC) of each of the algorithms proposed, when considering all the samples in the database already described.

The Equal Error Rate [27] is obtained as follows:

- Template Creation: The template of each user is made up of three of their authentic in-air signature samples. Then, $\mu$ is calculated.

- Analysis of authentic samples: The remaining four real samples of each user are used to evaluate whether the system grants or denies access to the authentic users. For each authentic sample, $\Psi / \mu$ is obtained when comparing the accessing gesture with the three gestures of the user template.

- Analysis of falsified samples: All the falsifying attempts trying to access the system will be used to evaluate whether the system is able or not to reject impostors. For each falsification trial, $\Psi / \mu$ is also obtained.

- Obtention of False Acceptance Rate $(F A R(\theta))$ and False Rejection Rate $(F R R(\theta)): F A R(\theta)$ and $F R R(\theta)$ are obtained in terms of $\theta$ as the \% of original samples that are over $\theta$ in case of False Rejection Rates and the \%
TABLE I

EER RESULTS WHEN NO NORMALIZATION IS MADE.

\begin{tabular}{|c|c|}
\hline Algorithm & EER (\%) \\
\hline LCS & 7.11 \\
\hline DTW & 3.23 \\
\hline ELCS & 3.34 \\
\hline EDTW & 2.80 \\
\hline
\end{tabular}

of falsified samples that are under $\theta$ in case of False Acceptance Rates. $F A R(\theta)$ and $F R R(\theta)$ are obtained for values of $\theta$ from 0 to $\max (\Psi / \mu)$ in 10000 steps.

- Obtention of Equal Error Rate (EER): EER is defined as the intersection value of the False Acceptance Rate and the False Rejection Rate.

Additionally, the performance of the biometric systems may be also represented by a Receiver Operating Characteristic figure (ROC) [28], where axis X represents False Matching Rate (FMR) and axis Y True Matching Rate (TMR). When Failure-to-acquire (FTA) rate is 0 (as in the experiments presented in this article, since the samples evaluated come from a closed database), it is verified that FMR=FAR and FNMR=FRR. Besides, FNMR is defined as FNMR=1-TMR. Consequently, when FTA=0, it is equivalent to represent a ROC figure within FMR vs. TMR and FAR vs. (1-FRR). Moreover, Equal Error Rate can be also deducted from the intersection of the ROC curve with the line where FAR=1FRR. Actually, this point corresponds to $1-E E R$. Therefore, a ROC figure represents the errors of the system when FRR or FAR are fixed.

\section{RESULTS}

The results of the analysis of the samples in the database utilizing the algorithms previously described are presented in this Section. These results are separated by the technique used to normalize the signals detailed in Section III-A.

Each normalization technique results will include the Equal Error Rate obtained as well as the ROC curve when analyzing the signals with the LCS, DTW, ELCS and EDTW algorithms.

1) "No normalization": When the acceleration signals of the performance of the signatures are not modified, the EER in Table I are obtained.

From this table, some conclusions can be extracted:

- Algorithms based on DTW obtain better results than those based on LCS.

- Euclidean approach improves the results in respect to obtaining the distance directly from the algorithms in both cases.

- An optimal $2.80 \%$ of EER is obtained.

In addition to this, Figure 1 represents the ROC curves of applying any of the algorithms previously presented.

2) "Maximum normalization": When each acceleration signal is divided by 2.5 the signals are normalized in the range $[-1,1]$, and the EER results in Table II are obtained for each distance. 
Fig. 1. ROC curves when no normalization is made.

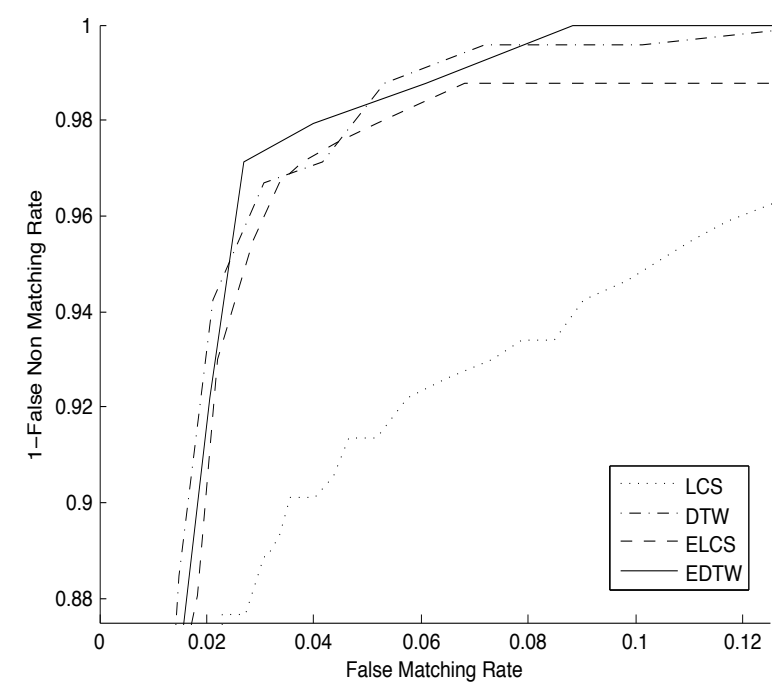

TABLE II

EER RESULTS WHEN “MAXIMUM NORMALIZATION” IS MADE.

\begin{tabular}{|c|c|}
\hline Algorithm & EER (\%) \\
\hline LCS & 12.76 \\
\hline DTW & 3.41 \\
\hline ELCS & 6.91 \\
\hline EDTW & 2.86 \\
\hline
\end{tabular}

TABLE III

EER RESULTS WHEN "MAXIMUM ON-SIGNAL NORMALIZATION” IS MADE.

\begin{tabular}{|c|c|}
\hline Algorithm & EER (\%) \\
\hline LCS & 13.18 \\
\hline DTW & 3.23 \\
\hline ELCS & 7.73 \\
\hline EDTW & 2.80 \\
\hline
\end{tabular}

From the results of this table, it can be deducted that "Maximum normalization" does not improve the results of no normalization approach. Actually, algorithms based on LCS present significantly worse EER values. However, DTW algorithms do not worsen too much (EER of EDTW is $0.06 \%$ more and EER of DTW is $0.18 \%$ more).

Besides, The ROC curves of the behaviour of the performance for each algorithm are presented in Figure 2.

3) "Maximum on-signal normalization": This normalization strategy divides each acceleration signal by its maximum absolute value, so not only all the signals are normalized in the range $[-1,1]$ but also, the maximum absolute value of the signal is 1 . Following this procedure, the EER in Table III are obtained.

As it can be inferred from the results of this table, "Maximum on-signal normalization" does not improve neither the
Fig. 2. ROC curves when "Maximum normalization" is made.

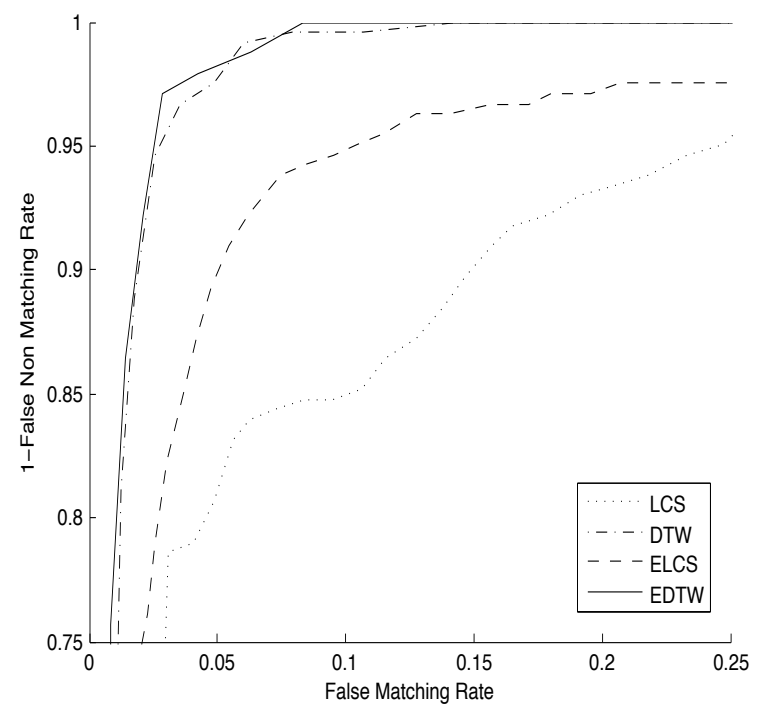

results of no normalization approach. As well as in "Maximum normalization", algorithms based on LCS present significantly worse EER values. However, utilizing DTW algorithms, the same EER results are obtained than when no normalization is performed.

ROC curves are shown in Figure 3.

Fig. 3. ROC curves when "Maximum on-signal normalization" is made.

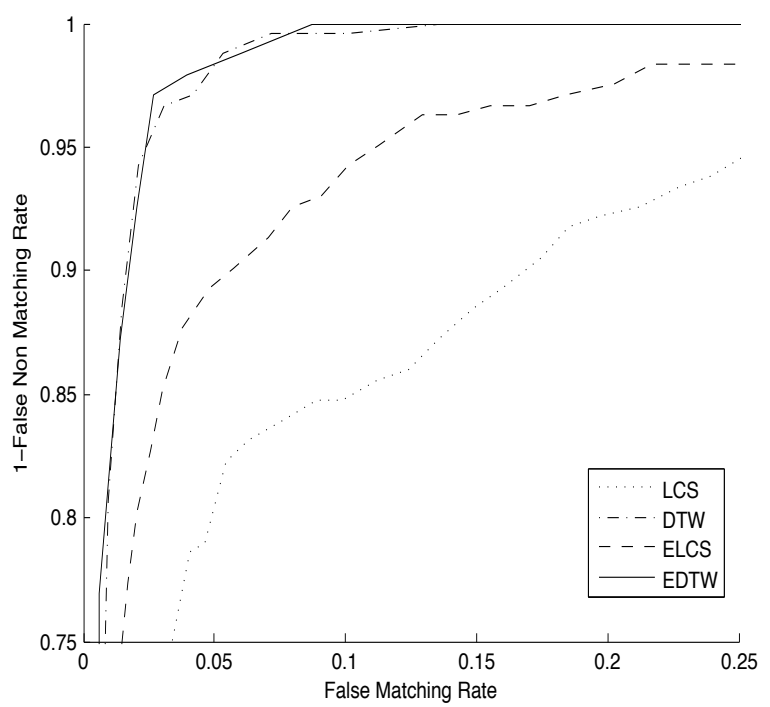

4) "Maximum-average normalization": In this case, the average of each signal is first subtracted, and then, the signal is divided by its maximum absolute value. Consequently, the signals are centered around 0 and belong to the range $[-1,-1]$. When this normalization method is applied, the EER values in Table IV are resulted.

The results of this Table mean that this method of normalization is absolutely not recommended for the analysis of acceleration signals with the temporal distance algorithms 
TABLE IV

EER RESULTS WHEN “MAXIMUM AVERAGE NORMALIZATION” IS MADE.

\begin{tabular}{|c|c|}
\hline Algorithm & EER (\%) \\
\hline LCS & 49.46 \\
\hline DTW & 48.09 \\
\hline ELCS & 47.25 \\
\hline EDTW & 48.15 \\
\hline
\end{tabular}

presented in this work, due to when normalization is performed applying this procedure, values of signals are deeply modified, so alignment algorithms do not work properly.

Finally, Figure 4 symbolizes the ROC curves of each of the algorithms presented when applying this normalization procedure.

Fig. 4. ROC curves when "Maximum-average normalization" is made.

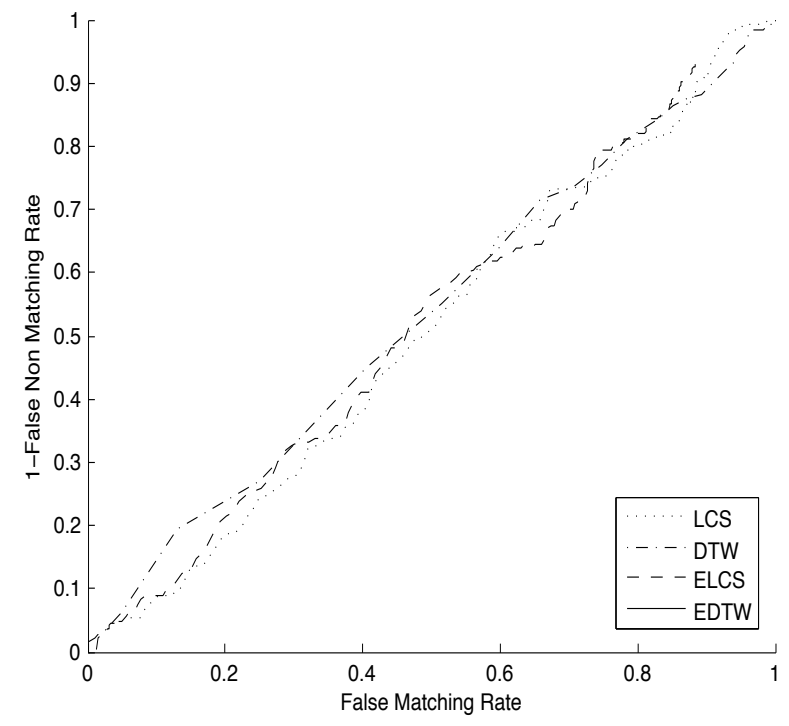

\section{CONCLUSION}

In this article a verification technique for mobiles embedding an accelerometer has been presented. Users are authenticated by making their signature in the air while holding their mobile phone.

Several temporal signal analysis approaches have been introduced based on Longest Common Subsequence and Dynamic Time Warping algorithms. These algorithms have been adapted to acceleration signals, and also, a modification of them has been proposed based on perform an optimal alignment and calculate the Euclidean distance of the aligned signals.

In addition to this, four different signal normalization methods have been proposed and evaluated: No normalization, "Maximum normalization", "Maximum on-signal normalization" and "Maximum-average normalization".

The four distance algorithms of temporal signals proposed and the four normalization strategies described have been assessed analyzing a database of 50 users who have performed their signature in the air in front of a video camera. From these records, each signature has been tried to be forged by six different people.

The evaluation of each algorithm and normalization method has been performed calculating the Equal Error Rate and presenting the Receiver Operating Characteristic curves.

From these experiments the following ideas are concluded:

- Algorithms based on Dynamic Time Warping distances offer a higher performance in respect to those based on Longest Common Subsequences.

- Normalization of the signals is not required since none of the strategies studied improves the results of when no normalization is performed.

Future works will be focused in studying different multibiometric architectures in order to fuse the information of the three axis in an optimal manner.

\section{REFERENCES}

[1] J. Chirillo and S. Blaul, Implementing Biometric Security, 1st ed. Hungry Minds, Incorporated, 2003.

[2] Q. Tao and R. Veldhuis, "Biometric authentication for a mobile personal device," Mobile and Ubiquitous Systems, Annual International Conference on, vol. 0, pp. 1-3, 2006.

[3] Y. Ijiri, M. Sakuragi, and S. Lao, "Security management for mobile devices by face recognition," in Mobile Data Management, 2006. MDM 2006. 7th International Conference on, May 2006, pp. 49 - 49.

[4] D. ho Cho, K. R. Park, D. W. Rhee, Y. Kim, and J. Yang, "Pupil and iris localization for iris recognition in mobile phones," Software Engineering, Artificial Intelligence, Networking and Parallel/Distributed Computing, International Conference on \& Self-Assembling Wireless Networks, International Workshop on, vol. 0, pp. 197-201, 2006.

[5] D. Jeong, H.-A. Park, K. Park, and J. Kim, "Iris recognition in mobile phone based on adaptive gabor filter," in Advances in Biometrics, ser. Lecture Notes in Computer Science, D. Zhang and A. Jain, Eds. Springer Berlin Heidelberg, 2005, vol. 3832, pp. 457-463.

[6] H. A. Shabeer and P. Suganthi, "Mobile phones security using biometrics," Computational Intelligence and Multimedia Applications, International Conference on, vol. 4, pp. 270-274, 2007.

[7] N. Clarke and S. Furnell, "Authenticating mobile phone users using keystroke analysis," International Journal of Information Security, vol. 6, pp. 1-14, 2007.

[8] R. Bellman, Dynamic Programming. Princeton University Press, 1957.

[9] H. Sakoe, "Dynamic programming algorithm optimization for spoken word recognition," IEEE Transactions on Acoustics, Speech, and Signal Processing, vol. 26, pp. 43-49, 1978.

[10] D. Berndt and J. Clifford, Using Dynamic Time Warping to Find Patterns in Time Series, 1994, pp. 359-370. [Online]. Available: http://www.aaai.org/Library/Workshops/1994/ws94-03-031.php

[11] E. J. Keogh and M. J. Pazzani, "Derivative dynamic time warping," in In First SIAM International Conference on Data Mining (SDM2001, 2001.

[12] S. Salvadore and P. Chan, "FastDTW: Toward accurate dynamic time warping in linear time and space," in 3rd Workshop on Mining Temporal and Sequential Data, 2004.

[13] M. Bashir and J. Kempf, "Person authentication with rdtw based on handwritten pin and signature with a novel biometric smart pen device," in Computational Intelligence in Biometrics: Theory, Algorithms, and Applications, 2009. CIB 2009. IEEE Workshop on, 30 2009-april 2 2009, pp. $63-68$.

[14] A. Piyush Shanker and A. N. Rajagopalan, "Off-line signature verification using dtw," Pattern Recogn. Lett., vol. 28, pp. 1407-1414, September 2007. [Online]. Available: http://portal.acm.org/citation.cfm?id=1274199.1274423

[15] A. Akl and S. Valaee, "Accelerometer-based gesture recognition via dynamic-time warping, affinity propagation," in Acoustics Speech and Signal Processing (ICASSP), 2010 IEEE International Conference on, march 2010, pp. $2270-2273$. 
[16] D. M. Gavrila and L. S. Davis, "Towards 3-d model-based tracking and recognition of human movement: a multi-view approach," in In International Workshop on Automatic Face- and Gesture-Recognition. IEEE Computer Society, 1995, pp. 272-277.

[17] D. S. Hirschberg, "Algorithms for the longest common subsequence problem," J. ACM, vol. 24, pp. 664-675, October 1977. [Online]. Available: http://doi.acm.org/10.1145/322033.322044

[18] L. Bergroth, H. Hakonen, and T. Raita, "A survey of longest common subsequence algorithms," in String Processing and Information Retrieval, 2000. SPIRE 2000. Proceedings. Seventh International Symposium on, 2000 , pp. $39-48$.

[19] Y.-T. Tsai, "The constrained longest common subsequence problem," Information Processing Letters, vol. 88 , no. 4, pp. 173 - 176, 2003. [Online]. Available: http://www.sciencedirect.com/science/article/pii/S002001900300406X

[20] I.-H. Yang, C.-P. Huang, and K.-M. Chao, "A fast algorithm for computing a longest common increasing subsequence," Information Processing Letters, vol. 93, no. 5, pp. 249 - 253, 2005. [Online]. Available: http://www.sciencedirect.com/science/article/pii/S0020019004003242

[21] A. Flores-Mendez and M. Bernal-Urbina, "Dynamic signature verification through the longest common subsequence problem and genetic algorithms," in Evolutionary Computation (CEC), 2010 IEEE Congress on, july 2010, pp. $1-6$.

[22] C. Choi, J.-H. Ahn, and H. Byun, "Visual recognition of aircraft marshalling signals using gesture phase analysis," in Intelligent Vehicles Symposium, 2008 IEEE, june 2008, pp. $853-858$.

[23] M.-C. Kao and T.-H. Li, "Design and implementation of interaction system between humanoid robot and human hand gesture," in SICE Annual Conference 2010, Proceedings of, aug. 2010, pp. $1616-1621$.

[24] J. Guerra-Casanova, C. S. Avila, A. de Santos Sierra, G. B. del Pozo, and V. J. Vera, "A real-time in-air signature biometric technique using a mobile device embedding an accelerometer." in NDT (1), ser Communications in Computer and Information Science, F. Zavoral, J. Yaghob, P. Pichappan, and E. El-Qawasmeh, Eds., vol. 87. Springer, 2010, pp. 497-503.

[25] J. Guerra-Casanova, C. Sanchez-Avila, A. de Santos Sierra G. Bailador del Pozo, and V. Jara Vera, "Acceleration axis selection in biometric technique based on gesture recognition," in Intelligent Information Hiding and Multimedia Signal Processing (IIH-MSP), 2010 Sixth International Conference on, oct. 2010, pp. $360-363$.

[26] J. Guerra-Casanova, C. Sanchez-Avila, A. de Santos Sierra, and G. B. del Pozo, "Score optimization and template updating in a biometric technique for authentication in mobiles based on gestures," Journal of Systems and Software, vol. In Press, Accepted Manuscript, pp. -, 2011. [Online]. Available: http://www.sciencedirect.com/science/article/pii/S0164121211001427

[27] J. L. Wayman, A. K. Jain, D. Maltoni, and D. Maio, Biometric Systems: Technology, Design and Performance Evaluation. Secaucus, NJ, USA: Springer-Verlag New York, Inc., 2004.

[28] T. Fawcett, "An introduction to roc analysis," Pattern Recogn. Lett., vol. 27, pp. 861-874, June 2006. [Online]. Available: http://portal.acm.org/citation.cfm?id=1159473.1159475

\section{VITA}

Javier Guerra-Casanova, was graduated as Telecommunications Engineer by Universidad Politcnica de Madrid in 2008. $\mathrm{He}$ is currently working at the Research Group in Biometrics, Biosignals and Security (GB2S) of the Universidad Politécnica de Madrid, as R\&D engineer. He is currently a PhD student in ETSIT (Escuela Técnica Superior de Ingenieros de Telecomunicación). His $\mathrm{PhD}$ studies are focused on new biometric techniques based on behavioural characteristicss applied to mobile devices.

Dra. Carmen Sánchez-Ávila. She obtained his $\mathrm{PhD}$ in Mathematical Sciences in 1993, by the Universidad Politécnica de Madrid (UPM), being currently Associate Professor at UPM. She is now in leadership of the Research Group in Biometrics, Biosignals and Security (GB2S) of UPM, involved in project research and development concerning a broad range of applications, from Mobile Security Services based on Biometric till Fast Cryptographic Protocols, Secure Transmission of Large Packages of Data and Crypto-Biometric. She is an expert in Biometrics and Cryptography Security and member of SC37 Standardization Committee.

Dr. Gonzalo Bailador received his Ph.D. degree in Computer Science from University Politécnica de Madrid. He is currently working in the research group GB2S focused on applying pattern recognition techniques to the analysis of temporal signals and mass spectrometry data. His research interests include gesture recognition, gait recognition, odour identification and robotics.

Alberto de Santos Sierra, received the degree of Telecommunication Engineer in Escuela Tecnica Superior de Telecomunicación (ETSIT), finishing his studies in Vrije Universiteit Amsterdam with a Final Master Project based on Iris Recognition, in July, 2007. At present, he is working at the G2BS in topics related to Biometric Recognition Systems, Stress Biometry and Crypto-Biometric. He is currently a $\mathrm{PhD}$ Student in ETSIT focusing on Biometrics based on Hand Recognition and other physiological characteristics oriented to mobile devices. 